\title{
Monitoring of the plasma generated by a gas-puff target source
}

\author{
A. Torrisi, ${ }^{1, *}$ P. W. Wachulak, ${ }^{2}$ H. Fiedorowicz, ${ }^{2}$ and L. Torrisi ${ }^{3}$ \\ ${ }^{1}$ Nuclear Physics Institute, AS CR, Hlavnì 130, 25068 Husinec- $\check{e} e \check{z}$, Czech Republic \\ ${ }^{2}$ Institute of Optoelectronics, Military University of Technology, 00-908 Warsaw, Poland \\ ${ }^{3}$ Dipartimento di Scienze Fisiche-MIFT, Università di Messina, 98166 S. Agata, Messina, Italy
}

(Received 23 December 2018; published 14 May 2019)

\begin{abstract}
A 10-Hz repetition rate, Nd:YAG pulsed laser $(\lambda=1064 \mathrm{~nm}$, pulse energy of $0.69 \mathrm{~J}$, pulse duration of $3 \mathrm{~ns}$ ) irradiated a Xe double-stream gas-puff target source. The interaction gives rise to the formation of plasma and emission of soft $\mathrm{x}$-ray and extreme ultraviolet radiation. The produced plasma was investigated and characterized by a silicon carbide $(\mathrm{SiC})$ and a commercial silicon (Si) detector, applying different spectral filters. Some parameters such as the plasma stability and its evolution (time trace profile and pulse time duration) are presented and discussed, evidencing pros and cons of the employment of $\mathrm{SiC}$ detectors with respect to the traditional Si for laser-generated plasma diagnostic.
\end{abstract}

DOI: 10.1103/PhysRevAccelBeams.22.052901

\section{INTRODUCTION}

Silicon carbide $(\mathrm{SiC})$ is considered nowadays a very performant semiconductor due to its intrinsic properties such as the wide band gap, the high charge mobility, and the high thermal conductivity, allowing their operability at a high frequency, in the presence of visible light (VIS) and at a high temperature [1]. Because of their peculiar features, such as the rate of charge collection, the high resistance to radiation damage, and the possibility to operate at room temperature maintaining a low leakage current, $\mathrm{SiC}$ detectors are employed for the detection of neutrons [2], $\mathrm{x}$ rays [3], $\alpha$-particles [4], protons [5], and electrons [6]. Another advantage of $\mathrm{SiC}$, for ultraviolet (UV) applications, is their "blindness" to photons of energy $<3.2 \mathrm{eV}(\lambda>380 \mathrm{~nm})$ as well their strong atomic chemical bonding, which increases its radiation hardness. Moreover, due to their larger band gaps, the dark current of such devices is orders of magnitude lower than that of silicon photodetectors [7]. The suitability of high-quality epitaxial layers allows the fabrication of high-performance $\mathrm{SiC}$ detectors based on Schottky diodes and $p$ - $n$ junctions. The $\mathrm{SiC}$ detectors show many advantages with respect to silicon [8], such as their high efficiency, working temperature, and very short rise time, due to their particular design that leaves a fraction of the active region directly exposed to the impinging radiation [9]. Moreover, their high displacement energy (of

\footnotetext{
*Corresponding author. torrisi@ujf.cas.cz

Published by the American Physical Society under the terms of the Creative Commons Attribution 4.0 International license. Further distribution of this work must maintain attribution to the author(s) and the published article's title, journal citation, and DOI.
}

$25 \mathrm{eV}$ with respect to the $\mathrm{Si}$ of $15 \mathrm{eV}$ ) [10] makes them high temperature and radiation resistant, and their high electron and hole mobility allows us to use them for high-frequency counting mode operation. Having an energy resolution comparable to $\mathrm{Si}$ and being blind to VIS radiation, $\mathrm{SiC}$ detectors represent a valid alternative to employ for ion beam monitoring in accelerator systems. They can be used for ion beam analysis, Rutherford backscattering (RBS), and recoil detection analysis (ERDA), avoiding the need to acquire the background noise during the elastic, nuclear reaction analysis (NRA) and for particle-induced characteristic x-ray emission (PIXE), when ions and electrons interact with insulators, semiconductors, and visible fluorescent targets $[11,12]$. Moreover, $\mathrm{SiC}$ can be successfully employed as $\mathrm{x}$-ray detectors monitoring the electromagnetic deflection of energetic ions extracted from ion sources or during their mass-to-charge selection and focusing of accelerated ion beams or for diagnostics of accelerated and deflected energetic electron beams $[13,14]$.

Other applications consist of monitoring the interaction of accelerated beams with gaseous and solid targets even with a low atomic weight. For instance, they can be useful during acceleration stripping, when the ionic beam interacts with gaseous strippers or with thin graphite sheets, from which the soft $\mathrm{x}$ rays (SXR, $\lambda=0.1-10 \mathrm{~nm}$ ) are emitted. Placing such detectors in proximity to collimators and a pinhole, it is possible to employ them to center the accelerated beams. The interaction of accelerated ion beams with solid targets determines the production of soft and hard $\mathrm{x}$ rays, whose signal can be calibrated using fast $\mathrm{SiC}$ detectors, when the signal is proportional to the energy of the detected $x$ rays. Moreover, the new techniques of ion acceleration are using high-intensity pulsed lasers to generate nonequilibrium plasma from thin foils producing ion acceleration [15]. In these cases, the high-intensity SXR 
generated by a laser-matter interaction can be monitored using the presented $\mathrm{SiC}$ detectors instead of the traditional $\mathrm{Si}$ detectors which are fully invested by VIS light, generating high noise currents [16].

In this work, plasma generated by a gas-puff target source, in the range of SXR, extreme ultraviolet (EUV) $(\lambda=10-120 \mathrm{~nm})$, and UV $(\lambda=120-400 \mathrm{~nm})$ radiation [17], was investigated, comparing the behavior of a Si and a $\mathrm{SiC}$ detector and applying spectral filters to select radiation from different wavelength ranges. As already performed with other gas-puff targets [18], Xe gas has been employed to change the emission from the plasma source, in order to compare such detectors in terms of their linearity response, time evolution, and signal intensity.

\section{EXPERIMENTAL SETUP}

The setup employed to monitor the SXR and EUV emission from the laser-irradiated gas-puff target is depicted in Fig. 1.

The gas puff was produced by an electromagnetic valve, which injects the gases through a nozzle at different pressures, from 1 to $10 \mathrm{bar}$, allowing one to change the gas target density and, consequentially, the emission yield. A double-stream gas-puff target was irradiated by an $\mathrm{Nd}$ : YAG laser operating at the $1064 \mathrm{~nm}$ wavelength ( $3 \mathrm{~ns}$ pulse duration, $0.69 \mathrm{~J}$ pulse energy, and $0.1 \mathrm{~mm}$ of focal spot diameter), by injection into the vacuum of Xe as a working gas (emission in the wavelength range of $\lambda=1.5-27 \mathrm{~nm}$ ). The Xe gas jet (inner gas with high Z, $0.7 \mathrm{~mm}$ in diameter) was surrounded by a second flow of He gas (outer gas with low Z, $1.5 \mathrm{~mm}$ in diameter) from a concentric outer nozzle, in order to confine the Xe flux preventing the divergence of the working gas flow outside the central nozzle. More details about the source employed are described in Refs. [19-21]. An AXUV-HS1 Si photodiode (mentioned in the paper as HS1) from IRD, USA, with an active area of $0.05 \mathrm{~mm}^{2}$ and a capacitance of $20 \mathrm{pF}$, and a SiC detector, with an active area of $6.35 \mathrm{~mm}^{2}$ and a junction capacitance of $100 \mathrm{pF}$, were placed at a distance of 108 and $170 \mathrm{~mm}$, respectively, from the gas-puff source. The detection solid angle was $4.29 \mu \mathrm{sr}$ for HS1 and $219 \mu \mathrm{sr}$ for SiC. The

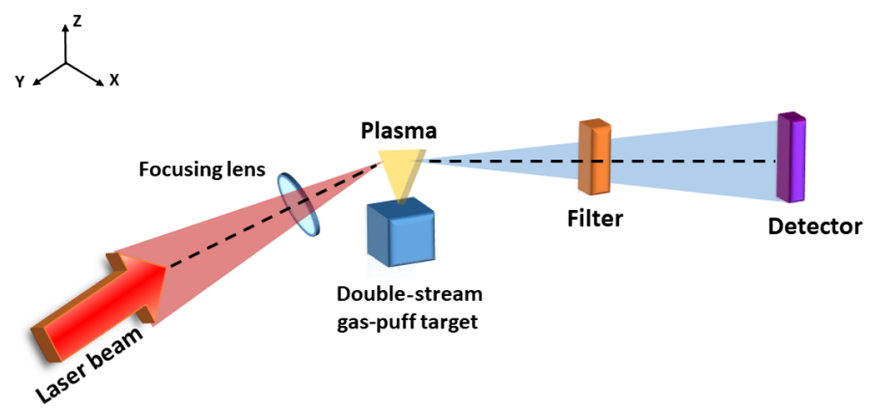

FIG. 1. Scheme of the experimental setup for EUV and SXR plasma emission monitoring. bias of the $\mathrm{SiC}$ and $\mathrm{Si}$ detectors was -10 and $-28 \mathrm{~V}$, respectively. The detectors were exposed to laser-generated plasma by the interaction of the laser beam with the gaspuff target. A fast storage oscilloscope TEKTRONIX-DPO 70404 (4-GHz bandwidth, $25 \mathrm{GS} / \mathrm{s}$ ) stored the signal responses of both detectors, as a function of the time. The $\mathrm{SiC}$ detector was interfaced to the oscilloscope through a capacitance of $100 \mathrm{pF}$, using an input impedance of $50 \Omega$, while the $\mathrm{Si}$ one through a capacitance 10 times lower.

The plasma was monitored employing $\mathrm{Al}, \mathrm{Ti}, \mathrm{Zr}$, and $\mathrm{CaF}_{2}$ filters, with a thickness of $750 \mathrm{~nm}, 500 \mathrm{~nm}, 250 \mathrm{~nm}$, and $5 \mathrm{~mm}$, respectively. Such filters were used with both detectors to select radiation from different wavelength ranges, the Ti filter in the range of $\lambda=0.1-6 \mathrm{~nm}$, the $\mathrm{Zr}$ filter in the range of $\lambda=8-18 \mathrm{~nm}$, the $\mathrm{Al}$ filter in the range of $\lambda=16-60 \mathrm{~nm}$, and, finally, the $\mathrm{CaF}_{2}$ filter in the range of $\lambda=180-300 \mathrm{~nm}[22,23]$. Figure 2(a) shows the detection efficiency $(\varepsilon)$ of the detectors employed [6, 24], while in Fig. 2(b) the transmissions $(\tau)$ of the used filters are depicted. Each detector works properly in a wavelength region dependent on the detection efficiency and on the filter transmission, and their response is proportional to the product $\varepsilon \tau$. Figure 2(c) shows, instead, the product of the detection efficiency of both detectors by the effective transmission of each applied filter. The comparison shows that the $\mathrm{SiC}$ detector has higher $(\varepsilon \cdot \tau)$ values as a function of the wavelength with respect to the $\mathrm{Si}$ and a better response to the lower wavelengths.

\section{EXPERIMENTAL RESULTS}

\section{A. Time evolution of the plasma}

The first study was devoted to the time evolution of the plasma. The plasma signal was monitored as a function of the time for both detectors. Each plot corresponds to a single pulse of radiation from the source averaged over ten measurements. In Figs. 3(a) and 3(b), the signal from HS1 (a) and $\mathrm{SiC}$ (b) detectors is depicted vs the time for Xe gas at a pressure of 10 bar for Xe and 6 bar for the outer $\mathrm{He}$ gas, employing different filters. Comparing the plots, it is possible to observe that the highest (peak) signal for HS1 detector was acquired employing Ti filter [Figs. 3(a) and 3(b)], while the higher signal for the $\mathrm{SiC}$ detector was obtained using the $\mathrm{Zr}$ filter. This results because $\mathrm{Zr}$ filter transmits in a range of $8-18 \mathrm{~nm}$ and also because the $\mathrm{Xe}$ gas emission is higher from $\sim 11 \mathrm{~nm}$ onward. In other words, the signal acquired was higher in the SXR range for the $\mathrm{Si}$ detector acquisition and in the EUV range for the $\mathrm{SiC}$ acquisition one.

In both cases, the signal is lower in the longer wavelength EUV range, obtained employing the $\mathrm{Al}$ filter, and in UV-VIS range, obtained employing the $\mathrm{CaF}_{2}$ filter. Moreover, the signal amplitudes are reversed for $\mathrm{Ti}$ and $\mathrm{Zr}$ detectors. The SXR component is well detected with $\mathrm{SiC}$ when the $\mathrm{Zr}$ filter is employed [Fig. 3(a)]. Employing the $\mathrm{Ti}$ 
(a)

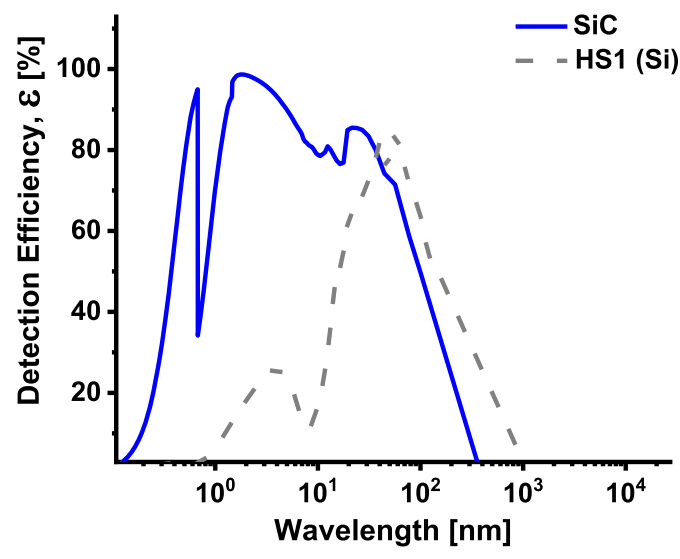

(b)

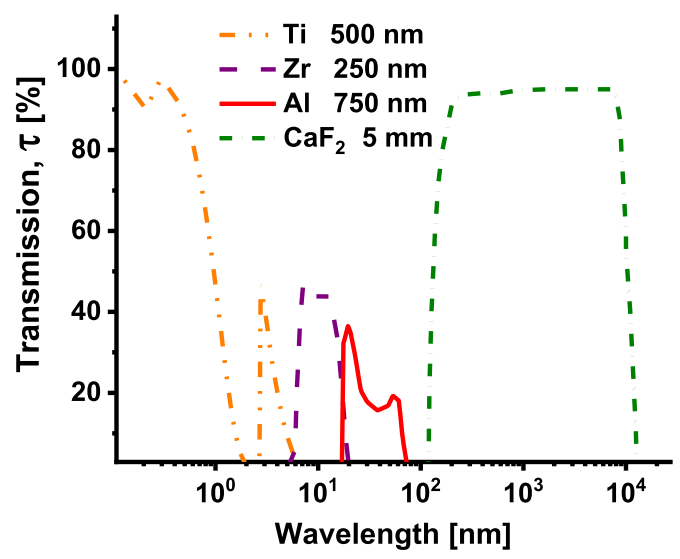

(c)

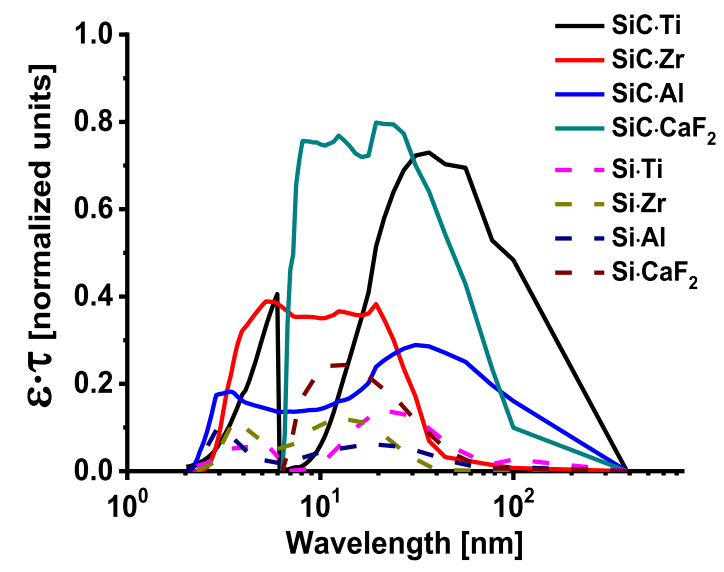

FIG. 2. (a) Detection efficiency $(\varepsilon)$ of AXUV-HS1 $\mathrm{Si}$ and $\mathrm{SiC}$ detectors. The $\mathrm{SiC}$ detection efficiency spans a range of $\lambda=0.1-350 \mathrm{~nm}$, while the Si detector cover the range of $\lambda=0.8-950 \mathrm{~nm}$. (b) Transmission $(\tau)$ of the filters employed at different wavelengths. Finally, (c) reports the contributions of each filter, for both detectors. Each filter allows one to select radiation from different wavelength ranges to modulate the plasma radiation in different wavelength ranges.

filter, instead, the SXR are strongly absorbed and only the EUV components are transmitted, detecting a high signal with the HS1 detector. It is possible to observe, as expected, that the plasma emits radiation in the SXR and EUV range for about 3 times the duration of the laser pulse, i.e., about 9-10 ns. In the case of the $\mathrm{SiC}$ detector, the pulse duration is affected by the time delay due to the $R C$ electronic circuit coupling the detector to the fast storage oscilloscope, of $5 \mathrm{~ns}(50 \Omega \cdot 100 \mathrm{pF})$, which enhances the delay time for 10-15 ns. Thus, the expected $\mathrm{SiC}$ pulse duration should be of about $25 \mathrm{~ns}$ or more, in agreement with the experimental results [Fig. 3(a)]. The intrinsic $R C$ delay of the $\mathrm{SiC}$ detector, of about $0.5 \mathrm{~ns}$, practically does not influence the total delay. The $\mathrm{Si}$ detector is faster with respect $\mathrm{SiC}$, because the total coupling electronics and intrinsic $R C$ give a time delay of the order of $1 \mathrm{~ns}$. Thus, a pulse signal is expected longer by about 3 times than the laser pulse duration, i.e., about $10 \mathrm{~ns}$, in good agreement with the experimental measurements [Fig. 3(b)]. Figures 3(c) and 3(d) show the timeintegrated signals of the HS1 (a) and $\mathrm{SiC}$ (b) detectors (yield $\mathrm{Y}_{\mathrm{HS} 1}$ and $\mathrm{Y}_{\mathrm{SiC}}$ measured in $\mathrm{V} \cdot \mathrm{ns}$ ) for $\mathrm{Xe}+\mathrm{Al}$, Ti, and $\mathrm{Zr}$, as a function of the gas pressures applied. The vertical error bars indicate the standard deviation of the integrated signal $( \pm \sigma)$, calculated for ten consecutive measurements acquired for the same gas pressure, for both detectors, while the horizontal error bars are related to the gas manometer accuracy of \pm 0.25 bar. We expected that increasing the gas pressure increases also its density and, consequently, the x-ray emission yield. In reality, instead, both detectors do not show a yield increment with the different filters, as depicted in Figs. 3(c) and 3(d). An explanation of this effect is that the photons transmitted by the employed filters arriving to the detectors are absorbed in the residual gas in the vacuum chamber, which increases with the gas pressure. Thus, although the gas pressure increases, the detector yield $(\mathrm{V} \cdot \mathrm{ns})$ does not increase significantly but remains almost constant.

Comparing Figs. 3(c) and 3(d), it is possible to conclude that, in the SXR-EUV range, the electrical SiC signal is only apparently higher than the $\mathrm{Si}$ one, because 
(a)

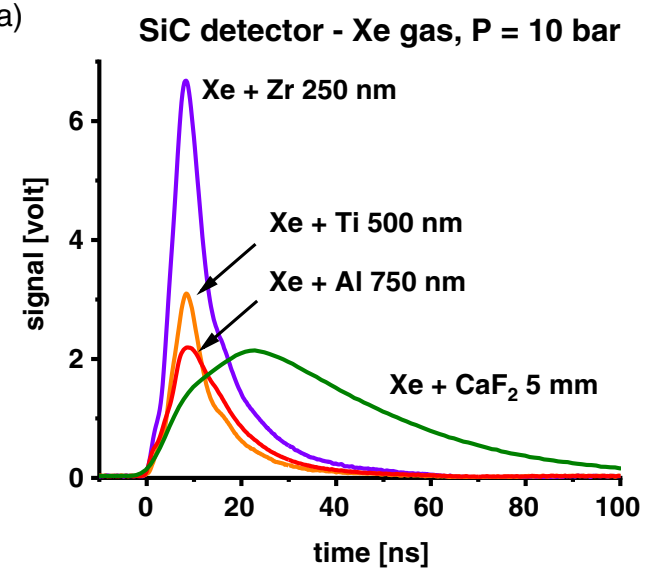

(c)

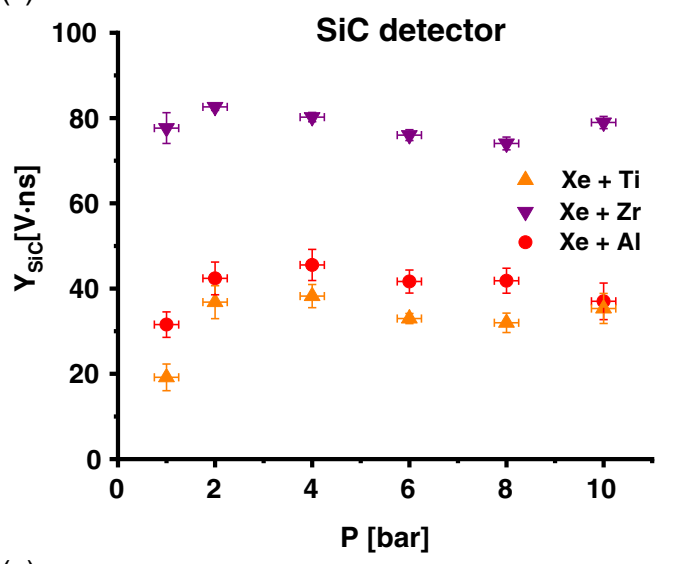

(e)

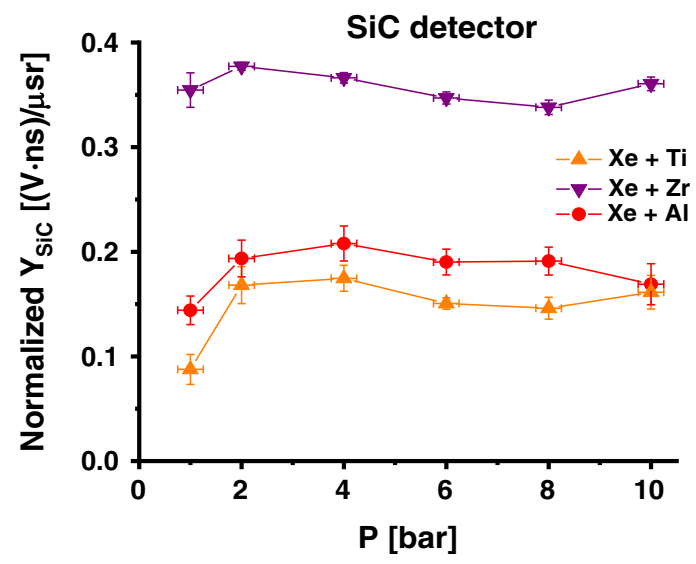

(b)

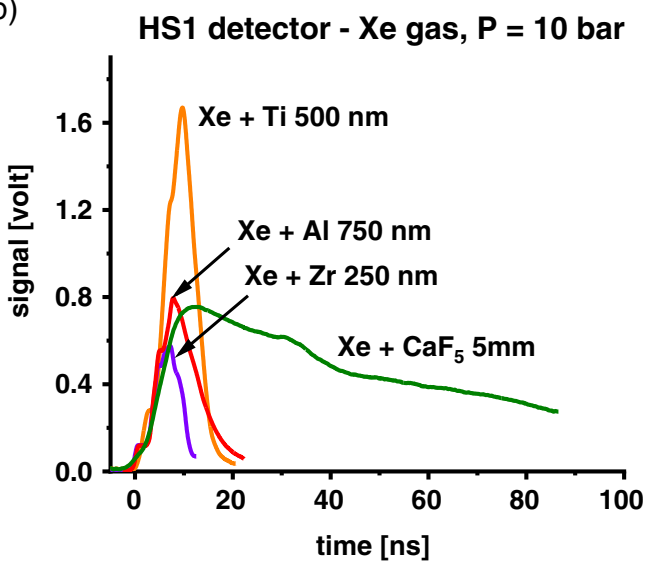

(d)

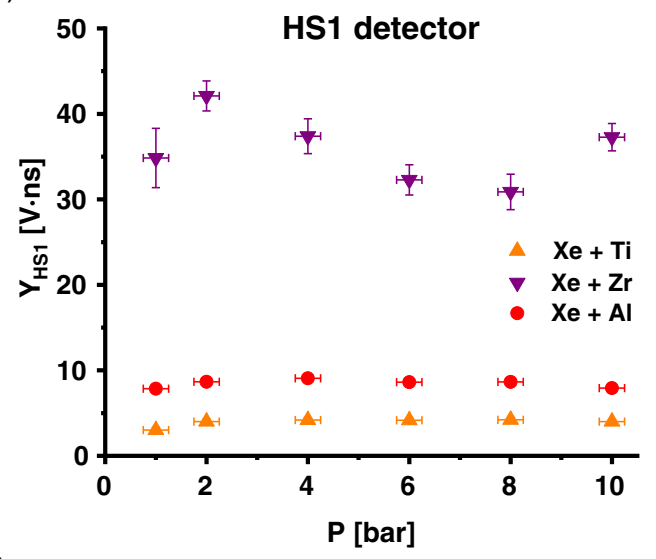

(f)

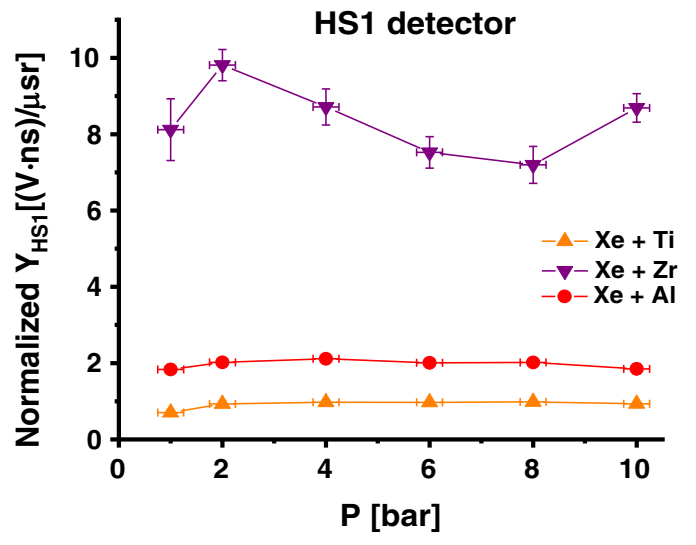

FIG. 3. Time traces of the detector signals for Xe plasma emission with HS1 (a) and SiC (b) for different wavelength ranges (from SXR to UV) selected by various filters. (c) and (d) show the integrated signal of HS1 and SiC as a function of the applied gas-puff backing pressures of Xe gas at a constant He pressure of 6 bar, while (e) and (f) show the integrated signal normalized in terms of a solid angle $(219 \mu$ sr for $\mathrm{SiC}$ and 4.29 for HS1).

normalizing to the solid detection angle is that of the $\mathrm{Si}$ signal to be higher in an absolute scale $(\mathrm{V} \cdot \mathrm{ns} / \mu \mathrm{sr})$, as shown in Figs. 3(e) and 3(f). One explanation of this evidence can be given by the larger area and electric capacitance of the $\mathrm{SiC}$ detector, which, in principle, would allow one to collect more charges with respect to the $\mathrm{Si}$ detector. However, since the energy to form an $e$ - $h$ pair in
$\mathrm{SiC}$ is $7.76 \mathrm{eV}$ while in $\mathrm{Si}$ it is only $3.63 \mathrm{eV}$, the highest charge is effectively collected by the detector HS1 (Si) and not by $\mathrm{SiC}$.

Even if from the comparison of Figs. 3(c) and 3(d) it seems that the time-integrated signals of $\mathrm{SiC}$ are higher than the HS1 ( $\mathrm{Si})$, normalizing the signal to the detection solid angle, it is exactly the opposite [Figs. 3(d) and 3(e)]. 

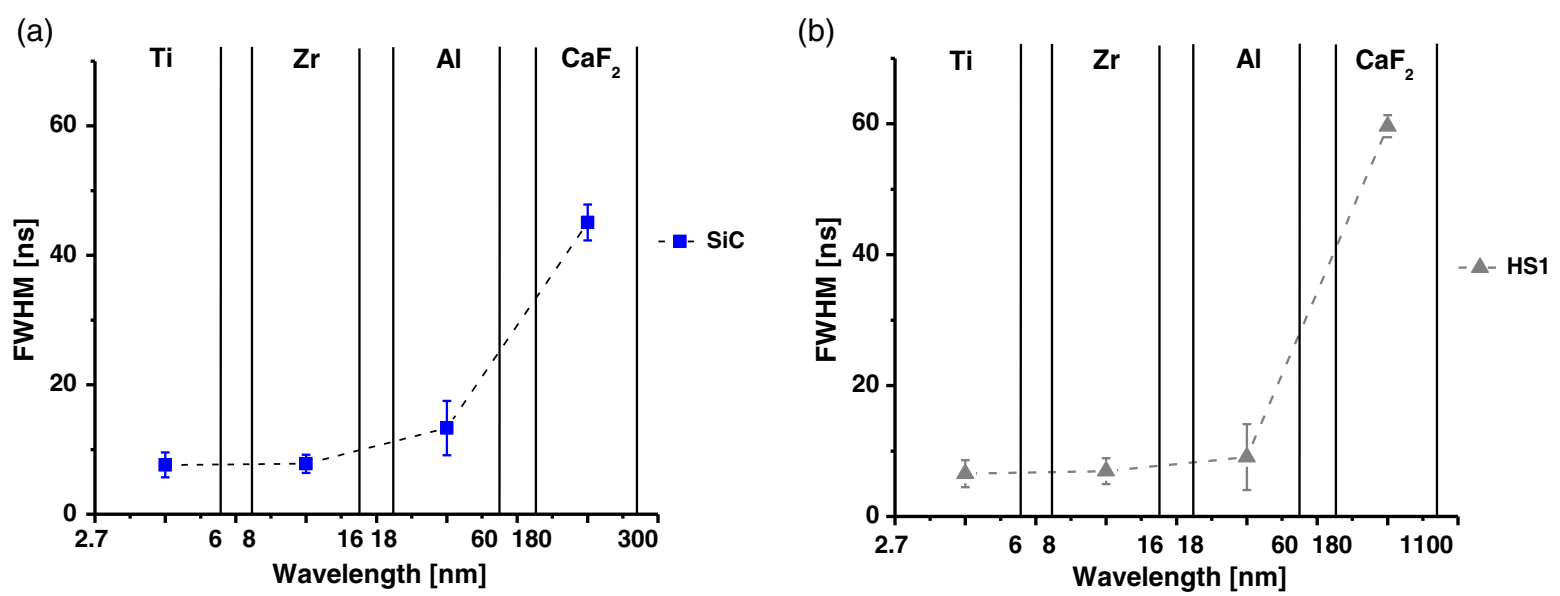

FIG. 4. Xe plasma emission evolution, for a gas-puff pressure constant, of 10 bar. Comparison of (a) SiC and (b) HS1 signals (in terms of FWHM) in different wavelength ranges limited by various filters. The $\mathrm{CaF}_{2}$ range spans a wavelength range of $180 \mathrm{~nm}$ to $10 \mu \mathrm{m}$, but the spectral sensitivity of HS1-Si and SiC detector with such a filter is from 180 to $300 \mathrm{~nm}$ (a) and from 180 to $1100 \mathrm{~nm}$ (b) wavelength, respectively. The statistical vertical error bar, over ten measurements, is reported as $20 \sigma$ for $\mathrm{Ti}, \mathrm{Zr}$, and $\mathrm{Al}$ filters and as $1 \sigma$ for the $\mathrm{CaF}_{2}$ filter, where $\sigma$ is the standard deviation.

Indeed, considering, for example, the signal [voltage $(\mathrm{V})]$ detected by HS1 at a pressure of 10 bar employing the Ti filter, we obtain a signal yield of about $4 \mathrm{~V} \cdot \mathrm{ns}$. Thus, the detection solid angle of Si being $4.29 \mu \mathrm{sr}$, the ratio between the yield and the solid angle will be $\sim 1 \mathrm{~V} \cdot \mathrm{ns} / \mu$ sr. Taking into account the same signal detected with the $\mathrm{SiC}$, we obtain from the signal yield of $35 \mathrm{~V} \cdot \mathrm{ns}$ and the solid angle of $219 \mu \mathrm{sr}$ a much smaller ratio, of $\sim 0.16 \mathrm{~V} \cdot \mathrm{ns} / \mu \mathrm{sr}$. This demonstrates that the $\mathrm{Si}$ shows higher sensitivity to the investigated wavelength range with respect to the $\mathrm{SiC}$.

\section{B. Measurement of the signal intensity}

By measuring the full width at half maximum (FWHM) of the detector peaks from time traces in Figs. 4(a) and 4(b), it was possible to determine the relationship between the detector signal duration and the plasma radiation emissions in different wavelength ranges, as depicted in Figs. 4(a) and 4(b). The statistical error, over ten measurements, is reported as $20 \sigma$ for all the filters employed, except for $\mathrm{CaF}_{2}$, where the $1 \sigma$ was taken into account, where $\sigma$ is the standard deviation. Plasma is produced during the laser-gas interaction at which it has the maximum temperature and density, emitting radiation from shorter wavelengths (SXR and high-energy EUV, selected with the use of $\mathrm{Ti}$ and $\mathrm{Zr}$ filters, respectively). After the laser pulse, of $3 \mathrm{~ns}$ duration, the plasma expands and cools down in the vacuum, emitting in the lower energy ranges of EUV (selected by using the $\mathrm{Al}$ filter) and UV-VIS $\left(\mathrm{CaF}_{2}\right.$ filter). Further research will be conducted in order to investigate the long pulse duration obtained using the $\mathrm{CaF}_{2}$ filter extended up to 100 ns. For both detectors (but, in particular, for HS1, having the lower energy gap of $1.1 \mathrm{eV}$ with respect to the $3.3 \mathrm{eV}$ of $\mathrm{SiC}$ ), it is possible to observe a long signal tail that may be generated due to the partial UV radiation from the plasma transmitted through the sample or due to the induced luminescence [25].

Because of the detector properties, to the coupling electronic detector oscilloscope and to the plasma luminescence induced in the filters, the detector FHWM, in the SXR-EUV range, extends about 10 and $15 \mathrm{~ns}$ in the case of $\mathrm{Si}$ and $\mathrm{SiC}$, respectively, as already shown in the time profile plots represented in Figs. 4(a) and 4(b). The FWHM extends up to about $60 \mathrm{~ns}$ in $\mathrm{Si}$ and less, to about $45 \mathrm{~ns}$, in SiC. Such a difference can be explained, because the luminescence is mainly produced in the VIS range rather than the UV, whose radiation is more absorbed inside the filter itself. It has to be taken into account that the $\mathrm{CaF}_{2}$ filter transmits in the range from $180 \mathrm{~nm}$ to $10 \mu \mathrm{m}$, but the spectral sensitivity of $\mathrm{SiC}$ and $\mathrm{Si}$ detectors, employing this filter, is from 180 to $300 \mathrm{~nm}$ for $\mathrm{SiC}$ [Fig. 4(a)] and from 180 to $1100 \mathrm{~nm}$ for Si [Fig. 4(b)]. In addition, as expected, the FWHM of $\mathrm{CaF}_{2}$ is higher for the Si detector (HS1), which has high detection efficiency in the VIS range, keeping in mind that the $\mathrm{SiC}$ detector, instead, has high detection efficiency only in the UV and shorter wavelengths. Nevertheless, the long signal duration suggests that the UV fluorescence is so intensive to be partially transmitted and detected by SiC. This explains why the SiC, which is typically blind to the VIS light, detects this long signal tail.

\section{Detector linearity}

A final study regarded the linearity response. Changing the $\mathrm{Xe}$ gas pressure in a range of $2-10 \mathrm{bar}$, it was possible to change the photon emission yield, as described in Sec. III A. The experimental results are depicted in Fig. 5, showing the $\mathrm{SiC}$ detector signal $\mathrm{Y}_{\mathrm{SiC}}$ in terms of the time-integrated signal $(\mathrm{V} \cdot \mathrm{ns})$ as a function of the HS1 


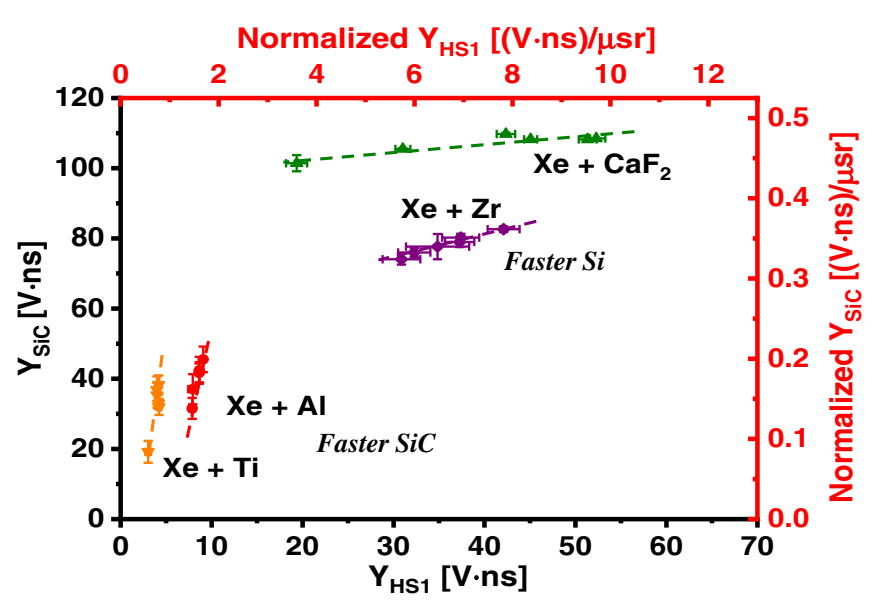

FIG. 5. Linearity test for Xe plasma emission. The plots report the $\mathrm{SiC}$ detector signal as a function of the HS1 detector in terms of time-integrated voltage and in terms of the normalized solid angles.

detector time-integrated signal $\mathrm{Y}_{\mathrm{HS} 1}$, for the different filters applied. The data are reported in double scale, in order to compare them to the normalized yield to the detection solid angle. The error bars (horizontal and vertical) indicate the standard deviation $\sigma$, referring to ten consecutive measurements, calculated as $\pm \sigma$.

The UV signal obtained with the $\mathrm{CaF}_{2}$ filter has a lower slope than the other data series, due to the plasma-induced high luminescence of the filter, emitting UV radiation at about $282 \mathrm{~nm}$ [26]. This is clearly shown in Fig. 5, where it is possible to observe that the HS1 detector has a much higher sensitivity to that range than the $\mathrm{SiC}$ detector for the far EUV or UV and VIS range (the signal from HS1 increases much faster than the $\mathrm{SiC}$ signal due to its high sensitivity to the visible light). On the contrary, $\mathrm{SiC}$ detection is faster on the SXR (Ti) and far EUV range (Al).

\section{CONCLUSIONS}

In this paper, different aspects of the laser-produced Xe plasma employing a double-stream gas-puff target were investigated employing a $\mathrm{SiC}$ detector and a commercial (Si) HS1 detector. In particular, the time evolution of the plasma, the relative signal intensity of the two detectors, and the linearity as a function of the Xe gas-puff pressures were studied. The presented $\mathrm{SiC}$ detectors can be employed to monitor plasma produced from compact laboratory sources and from large facilities such as accelerators and for transport of charge particle beams. They can be employed to analyze the ion-gas and ion-thin foil interactions in stripper systems producing SXR emission, for RBS, ERDA, NRA, and PIXE analysis, for electron and ion detection in the time-of-flight configuration, and for spectroscopy, monitoring the production of characteristic $\mathrm{x}$-ray emission, as reported widely in the literature $[6,7,8,9,11-16,21]$. SiC detectors present a significant advantage with respect to the traditional $\mathrm{Si}$ detector, due to the higher-energy band gap, which makes the detector less sensitive to the VIS light produced by the laserproduced plasma. Thus, $\mathrm{SiC}$ does not need filters to reduce the VIS radiation reaching the detector. Nevertheless, the used $\mathrm{SiC}$ structure has a very reduced metallic collector area $(25 \%)$ with respect to the total active uncovered area (75\%), as reported in the literature [3]. The high electronhole density in the small sensitive volume of the $\mathrm{SiC}$ detector, limited in depth by the diode active structure, which extends only up to $4 \mu \mathrm{m}$ and by the collector electrodes of only $3 \mu \mathrm{m}$ in width, suggests that recombination effects can be produced at high charge density collection. Moreover, the lower reverse current of $\mathrm{SiC}$, at room temperature, about 2 orders of magnitude lower than $\mathrm{Si}$, confers higher sensitivity to the UV and SXR detection. In addition, the higher displacement energy [10] reduces the crystal damage to the detector under high radiation doses. Finally, the higher melting point of the $\mathrm{SiC}$, with respect to the Si [26], permits us to use the detector with thinner active regions and to have high efficiency for $\mathrm{x}$ rays, electrons, and ions at high energy [27]. Nevertheless, SiC shows effects of less linearity and of possible saturation at high x-ray fluences with respect to Si. On the contrary, the $\mathrm{Si}$ detector has the advantage to be employed also for detection of VIS radiation and to collect a higher number of $e-h$ pairs for its lower-energy gap. The uniform metallic surface collection and the higher electron and hole mobilities in $\mathrm{Si}$ with respect to $\mathrm{SiC}$ allows us to collect very well the high charge densities produced by high photon fluences without nonlinearity and saturation effects. Further studies are in progress, in order to characterize other semiconductor detectors (in particular, $\mathrm{GaS}$ and diamond) using the plasma from different gas-puff targets [18].

\section{ACKNOWLEDGMENTS}

This work was supported by the Polish National Science Centre (Narodowe Centrum Nauki), Opus programs, Grant Agreements No. UMO-2015/17/B/ST7/03718 and No. UMO-2015/19/B/ST3/00435 and by the Grant Agency of the Czech Republic (Grantová agentura České republiky, GA CR) Project No. 19-02804S. We thank Dr. A. Sciuto (CNR-IMM, Catania) and Dr. M. Mazzillo (ST-Microelectronics, Catania) for the preparation and the employment of the $\mathrm{SiC}$ detector.

[1] J. B. Casady and R. W. Johnson, Status of silicon carbide ( $\mathrm{SiC}$ ) as a wide-bandgap semiconductor for high-temperature applications: A review, Solid State Electron. 39, 1409 (1996).

[2] L. Y. Liu, X. P. Ouyang, X. P. Zhang, J. L. Liu, J. L. Ruan, P. Jin, Z. N. Tian, L. C. Su, R. H. Huang, and S. Bai, A 4H silicon carbide based fast-neutron detection system with a 
neutron threshold of $0.4 \mathrm{MeV}$, Sens. Actuators, A 267, 547 (2017).

[3] A. Torrisi, P. W. Wachulak, A. Bartnik, T. Fok, W. Łukasz, H. Fiedorowicz, M. Mazzillo, A. Sciuto, and L. Torrisi, Calibration of $\mathrm{SiC}$ detectors for nitrogen and neon plasma emission using gas-puff target sources, IEEE Trans. Electron Devices 64, 1120 (2017).

[4] F. Moscatelli, Silicon carbide for UV, alpha, beta and x-ray detectors: Results and perspectives, Nucl. Instrum. Methods Phys. Res., Sect. A 583, 157 (2007).

[5] V. V Kozlovski, A. A. Lebedev, M. E. Levinshtein, S. L. Rumyantsev, and J.W. Palmour, Electrical and noise properties of proton irradiated $4 \mathrm{H}-\mathrm{SiC}$ Schottky diodes, J. Appl. Phys. 123, 024502 (2018).

[6] A. Cannavò, L. Torrisi, and L. Calcagno, SiC detector characterization for radiation emitted by laser-generated plasmas, J. Instrum. 11, C05008 (2016).

[7] L. Torrisi and A. Cannavò, SiC detectors to monitor ionizing radiations emitted from nuclear events and plasmas, Radiat. Eff. Defects Solids 171, 695 (2016).

[8] L. Torrisi and A. Cannavò, Silicon carbide for realization of 'telescope' ion detectors, IEEE Trans. Electron Devices 63, 4445 (2016).

[9] A. Sciuto, L. Torrisi, A. Cannavò, G. Ceccio, P. Musumeci, M. Mazzillo, and L. Calcagno, SiC interdigit detectors for post-accelerated ions generated by laser plasma, Vacuum 131, 170 (2016).

[10] R. Devanathan and W. J. Weber, Displacement energy surface in 3C and 6H SiC, J. Nucl. Mater. 278, 258 (2000).

[11] L. Torrisi, V. Havranek, M. Cutroneo, A. Mackova, L. Calcagno, A. Cannavò, and A. Torrisi, SiC detector for high helium energy spectroscopy, Nucl. Instrum. Methods Phys. Res., Sect. A 903, 309 (2018).

[12] G. Bertuccio, R. Casiraghi, A. Cetronio, C. Lanzieri, and F. Nava, A new generation of X-ray detectors based on silicon carbide, Nucl. Instrum. Methods Phys. Res., Sect. A 518, 433 (2004).

[13] A. Sciuto, G. D’Arrigo, S. Di Franco, M. Mazzillo, G. Franzo, L. Torrisi, and L. Calcagno, 4H-SiC detector in high photons and ions irradiation regime, IEEE Trans. Electron Devices 65, 599 (2018).

[14] S. Nida, A. Tsibizov, T. Ziemann, J. Woerle, A. Moesch, C. Schulze-briese, C. Pradervand, S. Tudisco, H. Sigg, O. Bunk, U. Grossner, and M. Camarda, Silicon Carbide X-ray Beam Position Monitors for Synchrotron Applications, J. Synchrotron Radiat. 26, 28 (2019).
[15] L. Torrisi, M. Cutroneo, A. Torrisi, L. Silipigni, G. Costa, M. Rosinski, J. Badziak, J. Wołowski, A. Zaraś-Szydłowska, and P. Parys, Protons accelerated in the target normal sheath acceleration regime by a femtosecond laser, Phys. Rev. Accel. Beams 22, 021302 (2019).

[16] A. Sciuto, L. Torrisi, A. Cannavò, M. Mazzillo, and L. Calcagno, Advantages and limits of $4 \mathrm{H}-\mathrm{SIC}$ detectors for high- and low-flux radiations, J. Electron. Mater. 46, 6403 (2017).

[17] The International Organization for Standardization, ISO 21348 Definitions of Solar Irradiance Spectral Categories, 2007.

[18] A. Torrisi, P. W. Wachulak, H. Fiedorowicz, and L. Torrisi, $\mathrm{SiC}$ detectors for evaluation of laser-plasma dynamics employing gas-puff targets, Nucl. Instrum. Methods Phys. Res., Sect. A 922, 250 (2019).

[19] H. Fiedorowicz, A. Bartnik, R. Jarocki, J. Kostecki, J. Krzywiński, J. Mikołajczyk, R. Rakowski, A. Szczurek, and M. Szczurek, Compact laser plasma EUV source based on a gas puff target for metrology applications, J. Alloys Compd. 401, 99 (2005).

[20] P. W. Wachulak, The novel gas puff targets for laser-matter interaction experiments, Rev. Sci. Instrum. 87, 091501 (2016).

[21] A. Torrisi, P. Wachulak, L. Torrisi, A. Bartnik, Ł. Węgrzyński, and H. Fiedorowicz, Plasma characterization of the gas-puff target source dedicated for soft x-ray microscopy using SiC detectors, Nukleonika 61, 139 (2016).

[22] CXRO-Center for X-ray optics database, http://henke.lbl .gov/optical_constants/.

[23] Newlight Photonics Web site, http://www.newlightphotonics .com/Optical-Windows-Rods/CaF2-Windows.

[24] Optodiode Corp., http://www.optodiode.com.

[25] W. Chen, L. Ma, R. Schaeffer, and R. Hoffmeyer, T. Sham, G. Belev, S. Kasap, and R. Sammynaiken, X-ray excited 459 optical luminescence of CaF2: A candidate for UV water 460 treatment, J. Phys. Conf. Ser. 619, 012047 (2015).

[26] Properties and Characteristics of Silicon Carbide, A. H. Rashed (Poco Graphite, Inc.), p. 1, http://www.poco.com/ Portals/0/Properties.and.Characteristics.of.Silicon.Carbide .pdf

[27] L. Torrisi, A. Sciuto, A. Cannavò, S. Di Franco, M. Mazzillo, P. Badalà, and L. Calcagno, SiC detector for sub-MeV alpha spectrometry, J. Electron. Mater. 46, 4242 (2017). 